\title{
Multifunctional Nanoparticulate Polyelectrolyte Complexes
}

\author{
Sean M. Hartig, ${ }^{1,2}$ Rachel R. Greene, ${ }^{1,3}$ Jayasri DasGupta, ${ }^{3}$ Gianluca Carlesso, ${ }^{3,4}$ Mikhail M. Dikov, \\ Ales Prokop, ${ }^{1}$ and Jeffrey M. Davidson ${ }^{3,6,7}$
}

Erratum to: Pharmaceutical Research

DOI 10.1007/s11095-007-9459-1

Two co-authors (DasGupta and Carlesso) and their affiliations were inadvertently omitted from the original article. The correct listing of authors and affiliations is shown in this erratum.

\footnotetext{
${ }^{1}$ Department of Chemical Engineering, Vanderbilt University, Nashville, Tennessee 37235-1604, USA.

${ }^{2}$ Present Address: Division of Pediatric Research, University of Texas MD Anderson Cancer Center, Houston, Texas 77030-4009, USA.

${ }^{3}$ Department of Pathology, Vanderbilt University School of Medicine, Nashville, Tennessee 37232-2562, USA.

${ }^{4}$ Department of Research, Medimmune Inc., One Medimmune Way, Gaithersburg, Maryland 20878, USA.

${ }^{5}$ Department of Medicine, Hematology/Oncology Division, Vanderbilt University School of Medicine, Nashville, Tennessee 37232-2562, USA.

${ }^{6}$ Research Service, VA Tennessee Valley Healthcare System, Nashville, Tennessee 37212-2637, USA.

${ }^{7}$ To whom correspondence should be addressed. (e-mail: jeff.davidson@vanderbilt.edu)
} 